\title{
Screening for pulmonary arterial hypertension in an unselected prospective systemic sclerosis cohort
}

\author{
Els Vandecasteele ${ }^{1}$, Benny Drieghe ${ }^{1}$, Karin Melsens ${ }^{2}$, Kristof Thevissen $^{3}$, \\ Michel De Pauw ${ }^{1}$, Ellen Deschepper ${ }^{4}$, Saskia Decuman ${ }^{2}$, Carolien Bonroy $^{5}$, \\ Yves Piette ${ }^{3}$, Filip De Keyser ${ }^{2,3}$, Guy Brusselle ${ }^{6}$ and Vanessa Smith ${ }^{2,3}$ \\ Affiliations: ${ }^{1}$ Dept of Cardiology, Ghent University Hospital, Ghent, Belgium. ${ }^{2}$ Dept of Internal Medicine, Ghent \\ University, Ghent, Belgium. ${ }^{3}$ Dept of Rheumatology, Ghent University Hospital, Ghent, Belgium. ${ }^{4}$ Biostatistics \\ Unit, Dept of Public Health, Ghent University, Ghent, Belgium. ${ }^{5}$ Dept of Laboratory Medicine, Ghent University \\ Hospital, Ghent, Belgium. ${ }^{6}$ Dept of Respiratory Medicine, Ghent University Hospital, Ghent, Belgium.
}

Correspondence: Els Vandecasteele, Dept of Cardiology, Ghent University Hospital, De Pintelaan 185, 9000 Ghent, Belgium. E-mail: els.vandecasteeledugent.be

@ERSpublications

Echocardiography remains a candidate first step in screening for PAH in an unselected systemic sclerosis population http://ow.ly/nuoh3096nRh

Cite this article as: Vandecasteele E, Drieghe B, Melsens K, et al. Screening for pulmonary arterial hypertension in an unselected prospective systemic sclerosis cohort. Eur Respir J 2017; 49: 1602275 [https:// doi.org/10.1183/13993003.02275-2016].

ABSTRACT Screening for pulmonary arterial hypertension (PAH) in systemic sclerosis (SSc) improves outcomes. The DETECT screening algorithm is recommended in a high-risk SSc subgroup. This study aims to compare prospectively the positive predictive value of screening using the DETECT algorithm and the 2009 European Society of Cardiology/European Respiratory Society (ESC/ERS) guidelines, and to compare their cost-effectiveness in an unselected, day-to-day SSc population. Post hoc, screening according to the 2015 ESC/ERS guidelines using echocardiographic parameters alone ("2015 echo screening") or combined with the DETECT algorithm ("2015 combined screening") in high-risk subjects was analysed.

195 consecutive SSc patients included in the Ghent University Hospital SSc cohort were screened using different algorithms.

The referral rate for right heart catheterisation was 32\% (63 out of 195 patients) (46/4/13/34/40 patients using the DETECT algorithm/2009 guidelines/both/2015 echo screening/2015 combined screening). Right heart catheterisation was performed in 53 patients (84\%) $(36(78 \%) /$ four $(100 \%) / 13(100 \%) / 28(82 \%) / 32$ (80\%) patients recommended by the DETECT algorithm/2009 guidelines/both/2015 echo screening/2015 combined screening). PAH was diagnosed in three patients (incidence $1.5 \% \cdot \mathrm{year}^{-1}, 95 \%$ CI $0.5-4.4$ ), in whom all algorithms recommended a right heart catheterisation. The positive predictive value was $6 \%$ (95\% CI 2-17\%; three out of 49 patients) for the DETECT algorithm, 18\% (95\% CI 6-41\%; three out of 17 patients) for the 2009 guidelines, $23 \%$ (95\% CI 8-50\%; three out of 13 patients) for both, $11 \%$ (95\% CI 4-27\%; three out of 28 patients) for the 2015 echo screening and 9\% (95\% CI 3-24\%; three out of 32 patients) for the 2015 combined screening. The cost was EUR224/80/90/112 per patient using the DETECT algorithm/2009 guidelines/2015 echo screening/2015 combined screening.

Echocardiography may remain a candidate first step for PAH screening in SSc.

This article has supplementary material available from erj.ersjournals.com

Received: Aug 082016 | Accepted after revision: Feb 062017

Conflict of interest: Disclosures can be found alongside this article at erj.ersjournals.com

Copyright OERS 2017 


\section{Introduction}

Systemic sclerosis (SSc) is an autoimmune connective tissue disease characterised by vasculopathy and fibrosis of the skin and visceral organs, resulting in major organ damage with high morbidity and mortality [1-3]. Currently, interstitial lung disease and pulmonary arterial hypertension (PAH) are the leading causes of disease-related mortality, and an elevated systolic pulmonary artery pressure (PAP) at baseline echocardiography is associated with reduced survival $[1,2,4]$.

The prevalence of PAH in SSc is $5-12 \%$ and the cumulative incidence at 15 years is $15 \%[5,6]$. Both the limited cutaneous SSc (LcSSc) and diffuse cutaneous SSc (DcSSc) subtypes are at risk for development of $\mathrm{PAH}$, and although the vast majority of new diagnoses of PAH occur after many years of Raynaud's phenomenon, PAH may occur early after diagnosis of SSc $[7,8]$. Screening programmes lead to earlier diagnosis, to earlier treatment and to a better prognosis [9-11]. In the current literature, no data are available on the prevalence of PAH in limited SSc (LSSc), a form of SSc without skin involvement, but with the presence of key prognostic markers to predict development of SSc $[12,13]$.

Annual screening using echocardiography in asymptomatic SSc patients is recommended by the 2009 expert consensus of the American College of Cardiology Foundation/American Heart Association and by the 2009 European Society of Cardiology/European Respiratory Society (ESC/ERS) guidelines on pulmonary hypertension $[5,14,15]$.

In a SSc subgroup at increased risk for PAH (SSc for $>3$ years since first non-Raynaud's phenomenon and diffusing capacity of the lung for carbon monoxide (DLCO) $<60 \%$ of the predicted value), the DETECT study introduced a two-step diagnostic algorithm to select SSc patients for right heart catheterisation (RHC) [16]. This prospective, multicentre cross-sectional study compared the accuracy of screening using the DETECT algorithm and the 2009 ESC/ERS guidelines, and was the first PAH study performing a RHC in all included high-risk SSc patients $[15,16]$. The referral rate for RHC was $62 \%$ versus $40 \%$ using the DETECT algorithm and 2009 guidelines, respectively, the number of missed PAH diagnoses $4 \%$ versus $29 \%$, the positive predictive value (PPV) $35 \%$ versus $40 \%$ and the negative predictive value (NPV) $98 \%$ versus $89 \%$. For screening by the DETECT algorithm, the high sensitivity ( $96 \%$ versus $71 \%$ ) was associated with a reduced specificity (48\% versus 69\%) compared with the 2009 guidelines [16].

The 2015 ESC/ERS guidelines recommend echocardiography for screening in asymptomatic SSc patients and annual screening combining echocardiography, DLCO and biomarkers may be considered in all SSc patients (as also described in the web addenda of KHANNA et al. [17]), and the use of the DETECT algorithm may be considered in a high-risk subgroup [5]. Recently, two prospective studies evaluated the accuracy of screening using the DETECT algorithm in a SSc population at risk for PAH and one study retrospectively evaluated the application of a modified DETECT algorithm in an unselected SSc population [18-20]. No prospective studies comparing the DETECT algorithm with the 2009 guidelines in an unselected, day-to-day population have been described in the literature to date.

The aim of our study was to evaluate the DETECT algorithm in an unselected, day-to-day SSc population. We compared, in a prospective manner, screening for PAH following the DETECT algorithm and the 2009 ESC/ERS guidelines ("2009 guidelines") in an unselected, day-to-day SSc cohort (including LSSc, LcSSc and DcSSc patients), and evaluated which of the two methods is the best performing as well as the cost-effectiveness of both methods. Additionally, post hoc, screening following the 2015 ESC/ERS guidelines using echocardiography alone ("2015 echo screening") or using a combination of echocardiography with the DETECT algorithm in the high-risk subgroup of our cohort ("2015 combined screening") was analysed.

\section{Methods}

All consecutive SSc patients included in the SSc unit of Ghent University Hospital (Ghent, Belgium) having their yearly SSc-specific visit between February 2015 and February 2016 were included. All patients fulfilled the preliminary classification criteria of the American College of Rheumatology (ACR) and/or the LeRoy and Medsger criteria for early SSc and/or the new ACR/European League Against Rheumatism (EULAR) SSc classification criteria [12, 21, 22]. Approval was obtained by the Ghent University Hospital Ethics Committee (amendment 2008/385) and all patients signed informed consent.

At the SSc-specific visit, all patients were evaluated by a standard SSc-specific evaluation also assessing for present/past telangiectasia and the extent of skin involvement, and were classified as LSSc, LcSSc or DcSSc [7]. Once classified as DcSSc, patients remained DcSSc irrespective of the current extent of skin involvement [6].

At the time of the SSc-specific visit (or within 2 months when impossible due to logistic problems), transthoracic echocardiography, a pulmonary function test, electrocardiography and a blood test were performed (see supplementary material for details) [23]. 
The DETECT algorithm was applied in our cohort using the DETECT calculator available at http:// detect-pah.com. When it was not possible to measure the peak tricuspid regurgitation velocity (TRV) accurately, "No measurable TRV" was chosen on the DETECT calculator [16].

The 2009 screening algorithm recommends referral for RHC in patients with a peak TRV $>3.4 \mathrm{~m} \cdot \mathrm{s}^{-1}$, a peak TRV $>2.8$ to $\leqslant 3.4 \mathrm{~m} \cdot \mathrm{s}^{-1}$ in combination with symptoms or a peak TRV $\leqslant 2.8 \mathrm{~m} \cdot \mathrm{s}^{-1}$ in combination with symptoms and additional echocardiographic variables suggestive for pulmonary hypertension as used in the original DETECT study (see supplementary material for details) $[15,16]$. When it was not possible to measure the TRV accurately, the 2009 screening algorithm was applied by the combination of symptoms and additional echocardiographic variables suggestive for pulmonary hypertension.

According to the 2015 ESC/ERS guidelines, RHC is recommended when patients have, based on echocardiographic findings, an intermediate or high risk for pulmonary hypertension defined as peak TRV $>2.8$ or $\leqslant 2.8 \mathrm{~m} \cdot \mathrm{s}^{-1}$ (or not measurable) in combination with additional echocardiographic variables suggestive for pulmonary hypertension from at least two out of the three different categories (see supplementary material for details). In addition to the described echocardiographic screening according to the 2015 ESC/ERS guidelines, screening using a combination of echocardiography in all patients with the DETECT algorithm in the high-risk subgroup (disease duration $>3$ years and DLCO $<60 \%$ pred) was evaluated [5].

RHC was performed within 3 months of the SSc-specific visit when the patient was recommended by the 2009 guidelines and/or by the DETECT algorithm. Pulmonary hypertension was defined as a mean PAP $\geqslant 25 \mathrm{mmHg}$, borderline elevated PAP as a mean PAP $21-24 \mathrm{mmHg}$ and no pulmonary hypertension as a mean PAP $\leqslant 20 \mathrm{mmHg}$. PAH was confirmed by RHC: mean PAP $\geqslant 25 \mathrm{mmHg}$ during RHC with pulmonary capillary wedge pressure (PCWP) $\leqslant 15 \mathrm{mmHg}$ in the absence of extensive interstitial lung disease (as defined by GoH et al. [24]). Pulmonary hypertension secondary to left heart disease (LHD) was defined as a mean PAP $\geqslant 25 \mathrm{mmHg}$ during RHC with PCWP $>15 \mathrm{mmHg}[5,15]$.

Exclusion criteria were pulmonary hypertension confirmed by prior RHC and the use of PAH-specific therapy.

The cost-effectiveness of the different screening algorithms in our cohort was evaluated by measuring the cost per patient. We choose the real cost per patient, without the costs for RHCs that were refused. The costs according to the Belgian RIZIV/INAMI (Rijksdienst voor Ziekte- en Invaliditeitsverzekering/Institut National d'Assurance Maladie Invalidité) nomenclature were used for all the investigations, except for N-terminal pro-brain natriuretic peptide (NT-proBNP) (without RIZIV/INAMI nomenclature) and for anti-centromere antibody, where the expected best local estimate of cost was calculated [25].

\section{Statistical analysis}

For descriptive purposes, absolute numbers with percentages are shown for nominal categorical variables and medians with interquartile ranges for continuous variables. The PPV is the probability that subjects with a positive screening test truly have PAH. For the PPV and the yearly incidence, Wilson's confidence intervals for a single proportion were measured using Wilson's score statistic confidence intervals [26]. The cost per patient for screening following the 2009 guidelines and the 2015 echo screening was calculated by the sum of (the cost for echocardiography for all included patients plus the cost for the performed RHCs) divided by the number of included patients. The cost per patient for screening following the DETECT algorithm was calculated by the sum of (the cost for the parameters included in step 1 of the DETECT algorithm for all included patients plus the cost for echocardiography in those patients where step 1 of the DETECT calculator recommended echocardiography plus the cost for the performed RHCs) divided by the number of included patients. The cost per patient for screening following the 2015 combined screening was calculated by the sum of (the cost for echocardiography for all included patients plus the cost for the parameters included in step 1 of the DETECT algorithm for all included high-risk patients plus the cost for the performed RHCs) divided by the number of included patients. Statistical analysis was performed with SPSS Statistics version 23.0 (IBM, Armonk, NY, USA), R version 3.2.3 (www.R-project.org) and PropCIs library version 0.2-5 (https://cran.r-project.org).

\section{Results}

Inclusion

Between February 2015 and February 2016, 210 patients had their yearly SSc-specific visit. After exclusion of 15 patients, 195 (93\%) were included in study. 12 patients had pulmonary hypertension on previous RHC ( $n=8$ PAH, $n=2$ pulmonary hypertension secondary to LHD and $n=2$ pulmonary hypertension secondary to pulmonary disease), two patients had conservative treatment for other diseases than PAH (malignancy and non-revascularisable coronary artery disease at advanced age) and for one patient, no NT-proBNP was measured. 


\section{Baseline characteristics}

Of the 195 included patients, 192 patients (98\%) fulfilled the LeRoy and Medsger criteria, 100 patients (51\%) fulfilled the ACR criteria, and 164 patients (84\%) fulfilled the new ACR/EULAR criteria. 156 patients $(80 \%)$ were female, with a median age of 54 (42-62) years and a median disease duration since first non-Raynaud's phenomenon of 69 (28-132) months. 21 patients (11\%) were classified as LSSc, 139 (71\%) as LcSSc and $35(18 \%)$ as DcSSc (table 1).

\section{Screening}

The referral rate of RHC was 32\% (63 out of 195 patients). 46 patients (73\%) were recommended by the DETECT algorithm alone, four patients $(6 \%)$ by the 2009 guidelines alone and 13 patients (21\%) by both. Step 1 of the DETECT calculator recommended echocardiography in 118 patients (61\%). Step 2 of the DETECT calculator recommended a RHC in 59 patients (30\%). Following the 2009 guidelines, a RHC was recommended in 17 patients (9\%), and post hoc in 34 patients (17\%) by the 2015 echo screening and 40 patients $(21 \%)$ by the 2015 combined screening.

Of the 63 patients recommended for RHC, 53 patients (84\%) also underwent a RHC: 36 of the patients (78\%) where RHC was recommended using the DETECT algorithm alone, four patients (100\%) using the 2009 guidelines alone, 13 patients (100\%) using both algorithms, and post hoc 28 patients (82\%) using the 2015 echo screening and 32 patients $(80 \%)$ using the 2015 combined screening. From the 10 patients who refused a RHC, eight patients were in New York Heart Association functional class I or II.

RHC was recommended in 19\% (four out of 21) of the LSSc patients, 32\% (45 out of 139) of the LcSSc patients and 40\% (14 out of 35) of the DcSSc patients. A RHC was performed in three, 39 and 11 patients, respectively (table 2 ).

\section{Results of the RHC}

Of the 53 patients in whom a RHC was performed, pulmonary hypertension was diagnosed in nine patients ( $n=3$ PAH, $n=5$ pulmonary hypertension secondary to LHD and $n=1$ pulmonary hypertension secondary to pulmonary embolism). In eight of those nine patients (89\%), a RHC was recommended by both screening algorithms and in one patient (11\%) diagnosed with pulmonary hypertension secondary to LHD, RHC was recommended by the DETECT algorithm alone. 30 patients (57\%) had no pulmonary hypertension and 14 patients (26\%) had borderline PAP (table 2).

Three patients were diagnosed with $\mathrm{PAH}$, making the incidence of PAH $1.5 \% \cdot \mathrm{year}^{-1}$ (95\% CI 0.5-4.4). RHC was recommended in the three patients by all screening algorithms and these three patients had LcSSc. The PPV was 6\% (95\% CI 2-17\%; three out of 49 patients) for the DETECT algorithm, 18\% (95\% CI 6-41\%; three out of 17 patients) for the 2009 guidelines, $23 \%$ (95\% CI 8-50\%; three out of 13 patients) for screening with both algorithms, and post hoc $11 \%$ (95\% CI 4-27\%; three out of 28 patients) for the 2015 echo screening and 9\% (95\% CI 3-24\%; three out of 32 patients) for the 2015 combined screening. RHC had been recommended by the DETECT algorithm in 13 out of the 14 patients (93\%) diagnosed with borderline PAP, whereas the 2009 guidelines only recommended RHC in four (29\%) of them, and both the 2015 echo screening and 2015 combined screening in 10 (71\%) of them.

TABLE 1 Baseline characteristics of the 195 SSc patients

\begin{tabular}{lcc} 
& Patients & Value \\
\hline Age years & 195 & $54(42-62)$ \\
Female/male & 195 & $156 / 39(80.0 / 20.0)$ \\
Presence of Raynaud's phenomenon & 195 & $191(97.9)$ \\
Disease duration since first presence of Raynaud's phenomenon months & $103(47-184)$ \\
Disease duration since first presence of non-Raynaud's phenomenon months & 191 & $69(28-131.5)$ \\
LSSc/LcSSc/DcSSc & 184 & $21(10.8) / 139(71.3) / 35(17.9)$ \\
NYHA I/II/III/IV & 195 & $59(30.3) / 119(61.1) / 17(8.7) / 0(0)$ \\
ACA & 195 & $83(42.6)$ \\
Scl70 & 195 & $19(9.7)$ \\
Peak TRV m-s ${ }^{-1}$ & 139 & $2.4(2.2-2.6)$ \\
FVC \% pred & 193 & $106(93-118.5)$ \\
DLco \% pred & 192 & $76(63.25-87)$
\end{tabular}

Data are presented as $\mathrm{n}$, median (interquartile range) or $\mathrm{n}(\%)$. LSSc: limited systemic sclerosis; LcSSc: limited cutaneous systemic sclerosis; DcSSc: diffuse cutaneous systemic sclerosis; NYHA: New York Heart Association; ACA: anti-centromere antibody; Scl70: anti-topoisomerase I antibody; TRV: tricuspid regurgitation velocity; FVC: forced vital capacity; DLCO: diffusing capacity of the lung for carbon monoxide. 
TABLE 2 Right heart catheterisation (RHC) recommended and performed by the different screening algorithms and the results of the RHC

RHC recommended by

2009 guidelines and/or DETECT algorithm

Both 2009 guidelines and DETECT algorithm

DETECT algorithm alone

2015 echo screening

2015 combined screening

$\begin{array}{lr}\text { Patients (LSSc; } & \text { RHC } \\ \text { LcSSc; DcSSc) } & \text { performed }\end{array}$

$63(4 ; 45 ; 14)$

$13(1 ; 11 ; 1)$

$4(0 ; 1 ; 3)$

$46(3 ; 33 ; 10)$

$34(2 ; 23 ; 9)$

$40(3 ; 27 ; 10)$
$4(100)$

$36(78)$

$28(82)$

$53(84)$

$13(100)$

32 (80)
Results of the RHC (LSSc; LcSSc; DcSSc)

No PH: $2(0 ; 2 ; 0)$

Borderline PAP: $3(0 ; 3 ; 0)$

$\mathrm{PAH}: 3(0 ; 3 ; 0)$

PH secondary to LHD: $4(1 ; 2 ; 1)$

$\mathrm{PH}$ secondary to pulmonary disease: $0(0 ; 0 ; 0)$

$\mathrm{PH}$ secondary to pulmonary embolism: $1(0 ; 1 ; 0)$ No PH: $3(0 ; 0 ; 3)$

Borderline PAP: $1(0 ; 1 ; 0)$

$\mathrm{PAH}: 0(0 ; 0 ; 0)$

$\mathrm{PH}$ secondary to LHD: $0(0 ; 0 ; 0)$

$\mathrm{PH}$ secondary to pulmonary disease: $0(0 ; 0 ; 0)$

$\mathrm{PH}$ secondary to pulmonary embolism: $0(0 ; 0 ; 0)$ No PH: $25(2 ; 21 ; 2)$

Borderline PAP: $10(0 ; 5 ; 5)$ $\mathrm{PAH}: 0(0 ; 0 ; 0)$

$\mathrm{PH}$ secondary to LHD: $1(0 ; 1 ; 0)$

$\mathrm{PH}$ secondary to pulmonary disease: $0(0 ; 0 ; 0)$

$\mathrm{PH}$ secondary to pulmonary embolism: $0(0 ; 0 ; 0)$ No $\mathrm{PH}: 10(1 ; 7 ; 2)$

Borderline PAP: $10(0 ; 6 ; 4)$ $\mathrm{PAH}: 3(0 ; 3 ; 0)$

$\mathrm{PH}$ secondary to LHD: $4(1 ; 2 ; 1)$

$\mathrm{PH}$ secondary to pulmonary disease: $0(0 ; 0 ; 0)$ $\mathrm{PH}$ secondary to pulmonary embolism: $1(0 ; 1 ; 0)$ No PH: $14(2 ; 9 ; 3)$

Borderline PAP: $10(0 ; 6 ; 4)$ $\mathrm{PAH}: 3(0 ; 3 ; 0)$

PH secondary to LHD: $4(1 ; 2 ; 1)$

$\mathrm{PH}$ secondary to pulmonary disease: $1(0 ; 1 ; 0)$

$\mathrm{PH}$ secondary to pulmonary embolism: $0(0 ; 0 ; 0)$

Data are presented as $\mathrm{n}$ or $\mathrm{n}(\%)$. LSSc: limited systemic sclerosis; LcSSc: limited cutaneous systemic sclerosis; DcSSc: diffuse cutaneous systemic sclerosis; PAP: pulmonary artery pressure; PH: pulmonary hypertension; PAH: pulmonary arterial hypertension; LHD: left heart disease. 2009 guidelines: 2009 European Society of Cardiology/European Respiratory Society (ESC/ERS) guidelines; 2015 echo screening: screening following the 2015 ESC/ERS guidelines using echocardiographic parameters; 2015 combined screening: screening following the 2015 ESC/ERS guidelines using the combination of echocardiographic parameters for all patients included in the cohort with the DETECT algorithm in the high-risk subgroup (with disease duration $>3$ years and diffusing capacity of the lung for carbon monoxide $<60 \%$ pred).

In 56 patients (29\%), no peak TRV was measurable. In eight of these 56 patients, a RHC was recommended by the DETECT algorithm. Four had no pulmonary hypertension, one was diagnosed with borderline PAP, one with pulmonary hypertension secondary to LHD and two refused to undergo a RHC.

\section{Subgroup analysis}

For 174 SSc patients with either LcSSc or DcSSc, the findings were similar to those in the total cohort. The PPV was 7\% (95\% CI 2-18\%; three out of 46 patients) for the DETECT algorithm, 19\% (95\% CI 7-43\%; three out of 16 patients) for the 2009 guidelines, 25\% (95\% CI 9-53\%; three out of 12 patients) for screening with both algorithms, and post hoc 12\% (95\% CI 4-29\%; three out of 26 patients) for the 2015 echo screening and 10\% (95\% CI 4-26\%; three out of 29 patients) for the 2015 combined screening.

\section{Cost-effectiveness}

In our cohort, the average cost per patient for screening for PAH was EUR80 using the 2009 guidelines, EUR224 using the DETECT algorithm, EUR90 using the 2015 echo screening and EUR112 using the 2015 combined screening (table 3).

Subgroup analyses are described in the supplementary material.

Adverse events

There were no adverse events (major bleeding, haemodynamic instability) reported after the RHCs. 


\begin{tabular}{|c|c|c|c|c|}
\hline & Price EUR & Patients n & Cost EUR & Cost per patient EUR \\
\hline \multicolumn{5}{|l|}{2009 guidelines } \\
\hline Echocardiography\# & 63.21 & 195 & 12325.95 & \\
\hline $\mathrm{RHC}^{\#}$ & 187.22 & 17 & 3182.74 & \\
\hline Total cost & & & 15508.69 & 79.53 \\
\hline \multicolumn{5}{|l|}{ DETECT algorithm } \\
\hline $\mathrm{FVC}^{\#}$ & 22 & 195 & 4290 & \\
\hline$D \mathrm{LCo}^{\#}$ & 47 & 195 & 9165 & \\
\hline NT-proBNPI & 11 & 195 & 2145 & \\
\hline Serum urate & 0.48 & 195 & 93.6 & \\
\hline Electrocardiography\# & 17.77 & 195 & 3465.15 & \\
\hline $\mathrm{ACA}^{\text {व }}$ & 40 & 195 & 7800 & \\
\hline Echocardiography ${ }^{\#}$ & 63.21 & 118 & 7458.78 & \\
\hline $\mathrm{RHC}^{\#}$ & 187.22 & 49 & 9173.78 & \\
\hline Total cost & & & 43591.31 & 223.55 \\
\hline \multicolumn{5}{|l|}{2015 echo screening } \\
\hline Echocardiography\# & 63.21 & 195 & 12325.95 & \\
\hline $\mathrm{RHC}^{\#}$ & 187.22 & 28 & 5242.16 & \\
\hline Total cost & & & 17568.11 & 90.09 \\
\hline \multicolumn{5}{|l|}{2015 combined screening } \\
\hline $\mathrm{FVC}^{\#}$ & 22 & 26 & 572 & \\
\hline$D \mathrm{LCO}^{\#}$ & 47 & 26 & 1222 & \\
\hline NT-proBNPף & 11 & 26 & 286 & \\
\hline Serum urate $\#$ & 0.48 & 26 & 12.48 & \\
\hline Electrocardiography\# & 17.77 & 26 & 462.02 & \\
\hline $\mathrm{ACA}^{\text {q }}$ & 40 & 26 & 1040 & \\
\hline Echocardiography & 63.21 & 195 & 12325.95 & \\
\hline $\mathrm{RHC}^{\#}$ & 187.22 & 32 & 5991.04 & \\
\hline Total cost & & & 21911.49 & 112.37 \\
\hline
\end{tabular}

RHC: right heart catheterisation; FVC: forced vital capacity; DLCO: diffusing capacity of the lung for carbon monoxide; NT-proBNP: N-terminal pro-brain natriuretic peptide; ACA: anti-centromere antibody; RHC: right heart catheterisation. 2009 guidelines: 2009 European Society of Cardiology/European Respiratory Society (ESC/ERS) guidelines; 2015 echo screening: screening following the 2015 ESC/ERS guidelines using echocardiographic parameters; 2015 combined screening: screening following the 2015 ESC/ERS guidelines using the combination of echocardiographic parameters for all patients included in the cohort with the DETECT algorithm in the high-risk subgroup (with disease duration $>3$ years and DLCO $<60 \%$ pred).

\#: cost price for the investigation according to the Belgian RIZIV/INAMI (Rijksdienst voor Ziekte- en Invaliditeitsverzekering/Institut National d'Assurance Maladie Invaliditél nomenclature; ": according to the expected best local estimate of cost [25].

\section{Discussion}

In an unselected, day-to-day SSc population, including SSc patients both with and without skin involvement, we compared prospectively screening for PAH by the DETECT algorithm and the 2009 ESC/ERS guidelines, and post hoc by the 2015 ESC/ERS guidelines. The first major finding is that the referral rate for RHC was 30\% (59 out of 195 patients) using the DETECT algorithm, 9\% (17 out of 195 patients) using the 2009 guidelines, 7\% (13 out of 195 patients) using both algorithms, and post hoc 17\% (34 out of 195 patients) by the 2015 echo screening and 21\% (40 out of 195 patients) by the 2015 combined screening. RHC was performed in $83 \%$ (49 out of 59 patients) of the SSc patients in whom the DETECT algorithm recommended RHC, in 100\% (17 out of 17 patients) when the 2009 guidelines did, and post hoc in $82 \%$ (28 out of 34 patients) using the 2015 echo screening and 80\% (32 out of 40 patients) using the 2015 combined screening. Second, three patients were diagnosed with PAH (incidence $1.5 \% \cdot y^{-1} \mathrm{r}^{-1}, 95 \%$ CI $0.5-4.4$ ). RHC was recommended by all the screening algorithms in these three patients. The PPV was $6 \%$ (95\% CI $2-17 \%$; three out of 49 patients) using the DETECT algorithm, 23\% (95\% CI 8-50\%; three out of 13 patients) for screening using the DETECT algorithm and the 2009 guidelines, $18 \%$ (95\% CI 6-41\%; three out of 17 patients) using the 2009 guidelines, and post hoc $11 \%$ (95\% CI 4-27\%; three out of 28 patients) using the 2015 echo screening and 9\% (95\% CI 3-24\%; three out of 32 patients) using the 2015 combined screening. The third finding is that 14 SSc patients were diagnosed with borderline PAP. RHC had been recommended by the DETECT algorithm in 13 of those 14 patients (93\%) and both 2015 screening algorithms in 10 (71\%) of them, whereas the 2009 guidelines only recommended RHC in four (29\%) of them. Finally, no LSSc patients were diagnosed with PAH. 
Comparing the 2009 guidelines and the DETECT algorithm in an unselected, day-to-day SSc population, we suggest that echocardiography, easily obtainable, as recommended in the 2015 guidelines may remain a major, first-step screening tool for PAH in unselected SSc patients and this is for several reasons. First, the choice of a screening algorithm depends on the ease of its application. A RHC was performed only in $83 \%$ of the SSc patients where the DETECT algorithm recommended RHC and in $100 \%$ of the SSc patients where the 2009 guidelines recommended RHC. As described in the 2015 ESC/ERS guidelines, a screening method should use tools that are noninvasive, reproducible, cost-effective and associated with a high NPV for the condition [5]. In our opinion, an additional criterion for a good screening tool is the fast availability of the test result, which might be an explanation for the lower performance of RHC in the DETECT group. In our centre, when possible, all examinations are planned on a multidisciplinary basis on the same day. At the end of that day, the results are discussed with the patient and RHC is planned when necessary. Unfortunately, the laboratory findings included in the DETECT algorithm were not available at the time of discussion, which meant that if a RHC was recommended by the DETECT algorithm, the patient had to be contacted by phone later to propose and schedule a RHC. Second, SSc can provoke cardiac involvement. It is known that symptomatic cardiac involvement has a poor prognosis and echocardiography may be useful for earlier diagnose of cardiac failure in order to start conventional treatment [27]. Finally, the choice of the screening algorithm also depends on its cost-effectiveness. In our cohort, the average cost per patient for screening for PAH was EUR224/80/90/112 using the DETECT algorithm/2009 guidelines/2015 echo screening/2015 combined screening [25]. The facts that the DETECT algorithm recommends an additional echocardiography in the majority of the patients and that RHC was recommended by all algorithms for the three patients diagnosed with $\mathrm{PAH}$ also strengthen the belief that echocardiography remains an important first screening tool. However, since echocardiography does not routinely report the right atrial area, peak TRV and the additional echocardiographic parameters suggestive for pulmonary hypertension from the 2015 guidelines, the echocardiography should be performed and interpreted by clinicians with experience in the field of pulmonary hypertension.

Many of the SSc patients with borderline PAP have a mild stage of pulmonary vasculopathy [28]. Although patients with borderline PAP do not need any PAH-specific treatment, the 2015 ESC/ERS guidelines recommend close monitoring of these patients to detect the possible progression into pulmonary hypertension [5]. In our cohort, the DETECT algorithm recommended a RHC in $93 \%$ of the patients diagnosed with borderline PAP and the 2015 screening algorithms recommended a RHC in $71 \%$ of them, whereas the 2009 guidelines only recommended a RHC in $29 \%$ of them. The DETECT algorithm definitely might play a role in diagnosing borderline PAP.

No patients with LSSc were diagnosed with PAH. Not all the LSSc patients will develop SSc with skin involvement [13]. In our cohort, subgroup analysis of the SSc patients with either LcSSc or DcSSc revealed similar findings to those in the total cohort.

The main limitations of our study are the absence of data on sensitivity, specificity and NPV of the screening algorithms, and the fact that only three patients were diagnosed with PAH. RHC was not routinely performed in this unselected population, since RHC is an invasive investigation and guidelines recommend noninvasive screening tools in SSc before referral for RHC. This explains the absence of data on sensitivity, specificity and NPV. Only three patients were diagnosed with PAH and this low number might have contributed to the overlap of the confidence intervals for the PPV. One might argue that three PAH diagnoses are low, but together with the eight patients previously diagnosed with PAH in our cohort, the prevalence of PAH is $5.2 \%$ (11 out of 210 patients) and the incidence is $1.5 \% \cdot \mathrm{year}^{-1}$ (three out of 195 patients). Taking into account the number of included LSSc patients (11\%), our data are in accordance with the percentages described in the literature (prevalence 5-12\%, and a cumulative incidence $2 \%, 9 \%$ and $15 \%$, respectively, at 5, 10 and 15 years of follow-up) [5, 6]. One may argue that the DETECT algorithm should have been compared with the 2015 ESC/ERS guidelines prospectively, but since the purpose of our study was to evaluate the DETECT algorithm in an unselected SSc population, we compared the DETECT algorithm with the 2009 guidelines as in the original DETECT paper [16]. Moreover, the 2015 ESC/ERS guidelines were not available at the start of our study in February 2015 and after presentation of the 2015 ESC/ERS guidelines, we decided to analyse the data according to the new guidelines post hoc and not to change the study protocol.

\section{Conclusions}

In an unselected, day-to-day SSc population, screening for PAH was compared prospectively by the DETECT algorithm and the 2009 guidelines, and post hoc by the 2015 guidelines. The 1-year incidence of PAH was $1.5 \% \cdot y^{-1}{ }^{-1}$ and all the PAH patients were recommended for RHC by all screening algorithms. The PPV was $23 \%$ for the DETECT algorithm combined with the 2009 guidelines, $18 \%$ for the 2009 guidelines, 6\% for the DETECT algorithm, 11\% for the 2015 echo screening and 9\% for the 2015 combined screening algorithm. The referral rate for RHC was high by the DETECT algorithm, with a high 
number of false negatives. Echocardiography, safe, noninvasive and with immediate availability of the test result, may remain a major, first-step screening tool for PAH in unselected SSc patients. Other screening tools used by the DETECT algorithm are useful, particularly to detect borderline PAP.

\section{Acknowledgements}

V. Smith is Senior Clinical Investigator of the Research Foundation - Flanders (Belgium) (FWO) (1802915N).

Author contributions: Conception and design of the work: E. Vandecasteele, M. De Pauw and V. Smith. Data collection: all authors. Analysis of the data: E. Vandecasteele, B. Drieghe, M. De Pauw, V. Smith and E. Deschepper. Draft: E. Vandecasteele. Critical revision of the work: all authors. Final approval: all authors. All authors agree to be accountable for all aspects of the work in ensuring that questions related to the accuracy or integrity of any part of the work are appropriately investigated and resolved.

\section{References}

1 Steen VD, Medsger TA. Changes in causes of death in systemic sclerosis, 1972-2002. Ann Rheum Dis 2007; 66: 940-944.

2 Tyndall AJ, Bannert B, Vonk M, et al. Causes and risk factors for death in systemic sclerosis: a study from the EULAR Scleroderma Trials and Research (EUSTAR) database. Ann Rheum Dis 2010; 69: 1809-1815.

3 Decuman S, Smith V, Verhaeghe S, et al. Work participation and work transition in patients with systemic sclerosis: a cross-sectional study. Rheumatology 2012; 51: 297-304.

4 Hachulla E, Clerson P, Airo P, et al. Value of systolic pulmonary arterial pressure as a prognostic factor of death in the systemic sclerosis EUSTAR population. Rheumatology 2015; 54: 1262-1269.

5 Galie N, Humbert M, Vachiery JL, et al. 2015 ESC/ERS Guidelines for the diagnosis and treatment of pulmonary hypertension: The Joint Task Force for the Diagnosis and Treatment of Pulmonary Hypertension of the European Society of Cardiology (ESC) and the European Respiratory Society (ERS): Endorsed by: Association for European Paediatric and Congenital Cardiology (AEPC), International Society for Heart and Lung Transplantation (ISHLT). Eur Respir J 2015; 46: 903-975.

6 Nihtyanova SI, Schreiber BE, Ong VH, et al. Prediction of pulmonary complications and long-term survival in systemic sclerosis. Arthritis Rheumatol 2014; 66: 1625-1635.

7 LeRoy EC, Black C, Fleischmajer R, et al. Scleroderma (systemic sclerosis): classification, subsets and pathogenesis. J Rheumatol 1988; 15: 202-205.

8 Hachulla E, Launay D, Mouthon L, et al. Is pulmonary arterial hypertension really a late complication of systemic sclerosis? Chest 2009; 136: 1211-1219.

9 Humbert M, Yaici A, de Groote P, et al. Screening for pulmonary arterial hypertension in patients with systemic sclerosis: clinical characteristics at diagnosis and long-term survival. Arthritis Rheum 2011; 63: 3522-3530.

10 Hachulla E, Launay D, Yaici A, et al. Pulmonary arterial hypertension associated with systemic sclerosis in patients with functional class II dyspnoea: mild symptoms but severe outcome. Rheumatology 2010; 49: 940-944.

11 Phung S, Strange G, Chung LP, et al. Prevalence of pulmonary arterial hypertension in an Australian scleroderma population: screening allows for earlier diagnosis. Intern Med J 2009; 39: 682-691.

12 LeRoy EC, Medsger TA Jr. Criteria for the classification of early systemic sclerosis. J Rheumatol 2001; 28: 1573-1576.

13 Koenig M, Joyal F, Fritzler MJ, et al. Autoantibodies and microvascular damage are independent predictive factors for the progression of Raynaud's phenomenon to systemic sclerosis: a twenty-year prospective study of 586 patients, with validation of proposed criteria for early systemic sclerosis. Arthritis Rheum 2008; 58: 3902-3912.

14 McLaughlin VV, Archer SL, Badesch DB, et al. ACCF/AHA 2009 expert consensus document on pulmonary hypertension a report of the American College of Cardiology Foundation Task Force on Expert Consensus Documents and the American Heart Association developed in collaboration with the American College of Chest Physicians; American Thoracic Society, Inc.; and the Pulmonary Hypertension Association. J Am Coll Cardiol 2009; 53: 1573-1619.

15 Galie N, Hoeper MM, Humbert M, et al. Guidelines for the diagnosis and treatment of pulmonary hypertension: the Task Force for the Diagnosis and Treatment of Pulmonary Hypertension of the European Society of Cardiology (ESC) and the European Respiratory Society (ERS), endorsed by the International Society of Heart and Lung Transplantation (ISHLT). Eur Heart J 2009; 30: 2493-2537.

16 Coghlan JG, Denton CP, Grunig E, et al. Evidence-based detection of pulmonary arterial hypertension in systemic sclerosis: the DETECT study. Ann Rheum Dis 2014; 73: 1340-1349.

17 Khanna D, Gladue H, Channick R, et al. Recommendations for screening and detection of connective tissue disease-associated pulmonary arterial hypertension. Arthritis Rheum 2013; 65: 3194-3201.

18 Hao Y, Thakkar V, Stevens W, et al. A comparison of the predictive accuracy of three screening models for pulmonary arterial hypertension in systemic sclerosis. Arthritis Res Ther 2015; 17: 7.

19 Nagaraja V, Visovatti S, Gladue H, et al. Screening algorithm for pulmonary hypertension in systemic sclerosis comparison of predictive accuracy in three algorithms. Arthritis Rheum 2015; 67: Suppl. 10, 3139.

20 Soukup T, Pudil R, Kubinova K, et al. Application of the DETECT algorithm for detection of risk of pulmonary arterial hypertension in systemic sclerosis: data from a Czech tertiary centre. Rheumatology 2016; 55: $109-114$.

21 Subcommittee for Scleroderma Criteria of the American Rheumatism Association Diagnostic and Therapeutic Criteria Committee. Preliminary criteria for the classification of systemic sclerosis (scleroderma). Arthritis Rheum 1980; 23: 581-590.

22 van den Hoogen F, Khanna D, Fransen J, et al. 2013 classification criteria for systemic sclerosis: an American College of Rheumatology/European League Against Rheumatism collaborative initiative. Ann Rheum Dis 2013; 72: 1747-1755.

23 Alere. Triage ${ }^{\circledast}$ NT-proBNP Test. Product Insert. http://www.alere.com/en/home/product-details/triage-nt-probnp. html Date last accessed: October 17, 2016.

24 Goh NS, Desai SR, Veeraraghavan S, et al. Interstitial lung disease in systemic sclerosis: a simple staging system. Am J Respir Crit Care Med 2008; 177: 1248-1254. 
25 Bonroy C, Smith V, Van Steendam K, et al. The integration of the detection of systemic sclerosis-associated antibodies in a routine laboratory setting: comparison of different strategies. Clin Chem Lab Med 2013; 51: $2151-2160$.

26 Altman DG, Machin D, Bryant T, et al. Statistics with Confidence. 2nd edn. London, BMC Books, 2000.

27 Vandecasteele EH, De Pauw M, Brusselle G, et al. The heart and pulmonary arterial hypertension in systemic sclerosis. Acta Clin Belg 2016; 71: 1-18.

28 Visovatti SH, Distler O, Coghlan JG, et al. Borderline pulmonary arterial pressure in systemic sclerosis patients: a post-hoc analysis of the DETECT study. Arthritis Res Ther 2014; 16: 493. 